\title{
SISTEMA REGIONAL DE INNOVACIÓN PARA EL DESARROLLO TERRITORIAL: PROPUESTA DE MODELO CUÁDRUPLE HÉLICE EN COLOMBIA
}

\author{
SISTEMA REGIONAL DE INOVAÇÃO PARA O DESENVOLVIMENTO \\ TERRITORIAL: PROPOSTA DE MODELO EM COLÔMBIA
}

\author{
Bernardo Javier Pérez Castaño ${ }^{1}$ \\ Elba María Bermúdez Quintana²
}

\section{RESUMEN}

En este artículo se analizan las bases de un modelo de gestión de la innovación para el desarrollo territorial, que propicie las interacciones entre los agentes del Sistema Nacional de Ciencia, Tecnología e Innovación; modelo sistémico representado por una cuádruple hélice viva compuesta por la administración, la investigación, la empresa y la sociedad, que incorpora los ingredientes críticos actuales para la innovación, modelo centrado en el impacto tanto sobre el desarrollo y los objetivos estratégicos de la región, como del sistema productivo y del sistema científico tecnológico. En esencia, con el modelo sistémico se busca lograr incrementos sustanciales y sistemáticos en el valor agregado de productos y servicios, mediante la aplicación de la ciencia, la tecnología y la innovación, tanto en los procesos productivos como en los no productivos. Ello implica entrar en círculos virtuosos de enriquecimiento del conocimiento, mediante su utilización, pasando del conocimiento individual al conocimiento grupal, lo cual se traduce en tecnología, esto es conocimiento aplicado; de este, a su vez, pasando al conocimiento organizacional que conduzca a la innovación, para llegar finalmente al conocimiento interinstitucional, representado en la creación de valor para los actores que intervienen (Gobierno, Unidades de Investigación, Empresas y la Sociedad en general). De esta manera se busca la consolidación de una comunidad científica y empresarial más competitiva en mercados abiertos, dinamizando la relación Universidad - Empresa - Estado - Sociedad, para alcanzar resultados eficaces en temas de Ciencia, Tecnología e Innovación, con un impacto positivo en el desarrollo territorial.

Palabras clave: Desarrollo territorial. Creación de valor económico y social. Cistema regional de innovación. Innovación. Desarrollo tecnológico. Conocimiento.

\section{RESUMO}

Este artigo analisa as bases de um modelo de gestão da inovação para o desenvolvimento territorial, que propicie interações entre os agentes do Sistema Naiocnal de Ciência, Tecnologia e Inovação, modelo sistémico representado por uma hélica quádrupla composta pela administração, invetigação, empresa e sociedade, que incorpora os ingredientes críticos atuais para a invoação, modelo centrado no impacto tantao sobre o desenvolvimento e os objetivos estratégicos da região, como do sistema produtivo e do sistema científico tecnológico. Em essência, o modelo sistêmico procurará alcançar aumentos substanciais e sistemáticas em produtos e serviços de valor agregado através da aplicação da ciência, tecnologia e inovação, tanto em processos produtivos e não produtivos. Trata-se de introduzir círculos virtuosos de enriquecimento do conhecimento através do uso, pasando do conhecimento individual para o

\footnotetext{
${ }^{1}$ Universidad del Valle, Colômbia. Grupo de Investigación Previsión y Pensamiento Estratégico. Cl. 13 \#100-00, Cali, Valle del Cauca, Colômbia. E-mail: bernardoperezcastano@gmail.com

${ }^{2}$ Pontificia Universidad Javeriana, Colômbia. Facultad de Odontología. KR 7 \# 40-62, Cra. 7, Bogotá, Colômbia. E-mail: embq2012@gmail.com
} 
conhecimento do grupo, que se traduz em tecnología. Esse conhecimento é aplicado, e, por sua vez, a partir do conhecimento organizacional que leva à inovação, passa-se finalmente para o interconhecimento, representado na criação de valor para os jogadores envolvidos (governo, unidades de investigação, empresas e sociedade em geral). Assim, é consolidadda uma comunidade científica e empresarial mais competitiva na busca de mercados abertos, agilizando a relação entre universidade, empresa, Estado e sociedade, para alcançar resultados eficazes nas áreas da Ciência, Tecnologia e Inovação, com um impacto positivo no desenvolvimento territorial.

Palavras-chave: Desenvolvimento territorial. Criação de valor econômico e social. Sistema regional de inovacão. Inovacão. Desenvolvimento tecnológico. Conhecimento.

\section{Introducción}

Un sistema político y económico que no reparte beneficios a la mayoría de los ciudadanos no es sostenible a largo plazo. Con el tiempo, la fe en la democracia y la economía de mercado se erosionarán y se pondrá en tela de juicio la legitimidad de las instituciones y los acuerdos vigentes. (STIGLITZ, 2013)

En el artículo, se presentaran los conceptos y premisas básicas que consideramos se deben tener en cuenta para el diseño, construcción y consolidación de un Sistema Regional de Innovación (SRI) para el desarrollo territorial (DT) y lo llamaremos de manera integral como, SRIDT. La propuesta surge de diversas investigaciones realizadas durante estancias postdoctorales de uno de los autores, enriquecida con valiosos aportes complementarios del otro. Las principales características de nuestra propuesta, son:

- Es un modelo sistémico centrado en el impacto, tanto sobre el desarrollo y los objetivos estratégicos de la región, como del sistema productivo y del sistema científico tecnológico, en función del desarrollo territorial, en pro del bienestar de la sociedad.

- Al ser sistémico, debe propiciar las interacciones entre los agentes del Sistema Nacional de Ciencia, Tecnología e Innovación (CTeI): el estado, el sector productivo, el sector científico tecnológico y por supuesto, la sociedad.

- Se deben lograr incrementos sustanciales y sistemáticos en el valor agregado de productos y servicios, mediante la aplicación de la ciencia, la tecnología y la innovación, tanto en los procesos productivos, como en los no productivos.

- Debe facilitar el entrar en círculos virtuosos de enriquecimiento del conocimiento, mediante su utilización: 
pasando del conocimiento individual al conocimiento grupal, lo cual se traduce en tecnología, siendo esta un conocimiento aplicado;

pasando del conocimiento grupal al conocimiento institucional, que conduzca a la innovación;

$\checkmark$ finalmente pasando del conocimiento institucional al conocimiento interinstitucional, representado en la creación de valor para los actores que intervienen (la sociedad en general, el estado, el sector productivo y el sector científico tecnológico).

Las interacciones, a través de la formulación y la ejecución de proyectos, facilitarán la consolidación de una comunidad científica y empresarial más competitiva en mercados abiertos, dinamizando la relación Universidad - Empresa - Estado Sociedad para alcanzar resultados eficaces en temas de Ciencia, Tecnología e Innovación, con un impacto positivo en el desarrollo territorial.

El artículo se desarrolla presentando brevemente las premisas básicas y conceptos asociados con desarrollo territorial, innovación y sistema entrando luego en detalles sobre ellos.

- Se inicia por "el fin" que debe tener el sistema esto es, por "lo fundamental", el "para qué" un SRI; es decir, el impacto que se debe lograr, cual es el desarrollo territorial. "Desarrollo" es un concepto que en muchas ocasiones se interpreta solo como avance económico y justamente, queremos resaltar que el avance económico, no debería ser un fin en sí mismo, sino un medio para lograr avance y desarrollo social y es por ello, que lo asociamos con "Bienestar Territorial". Para ello, consideramos importante tener en cuenta que lo fundamental es el bienestar de las personas que allí habitan y el entorno que las enmarca y se basa, prioritariamente, en grandes pilares de desarrollo social como la salud, la educación, el modelo de interacción social, el tipo de gobierno y la calidad de gobernanza establecida, la cultura que predomina, el tipo de relación que se teje con el entorno y la clase de explotación económica, entre otros.

- De acuerdo con Boisier (1997), en no pocos países se vive actualmente la cruel paradoja de una simultánea aceleración del crecimiento económico y 
desaceleración del desarrollo, de un aumento de los índices macroeconómicos y de una disminución de los índices más concretos que miden convergencia, sea entre sectores, territorios, o personas. La idea de una cierta justicia social que acompañase al crecimiento, parece más y más lejana de la realidad. Marshall Wolfe hablaba del "esquivo desarrollo" en tanto que Douglass North, poco después de recibir el Nobel de Economía, señalaba en una entrevista de prensa que la receta neo-liberal para el "desarrollo era bien conocida y que a pesar de ello, muchos países que la seguían al pie de la letra durante décadas, no veían llegar el ansiado desarrollo.

Hablar de un sistema regional implica la delimitación geográfica de un espacio con unas connotaciones definidas, por lo tanto, cuando hablamos de desarrollo territorial, recalcamos que las regiones tienen particularidades específicas, diversas y cambiantes con el tiempo.

- Continuamos con el concepto de innovación, destacando que la entendemos como creación, tanto de valor económico como social, ya que, en muchas ocasiones, la creación de valor social no está contemplada o no se alcanza, y por ello al final, solo se llega al avance meramente económico, sin impactar de manera significativa en el bienestar territorial dado que, en muchos casos, solo unos pocos se benefician de ese logro económico e incluso pudiendo ir en detrimento del medio ambiente o de un colectivo en particular ${ }^{3}$. Adicionalmente, nuestra propuesta se basa en un concepto amplio de innovación, en función del incremento de la productividad, que involucra todo tipo de innovaciones, incluyendo el mejoramiento continuo.

- Finalizamos con el concepto de sistema como un conjunto de elementos, coordinados entre si y estructurados deliberadamente, para lograr propósitos. En nuestro caso, los elementos son los actores regionales, siendo estos: el estado, el sector científico tecnológico, el sector productivo y la sociedad; los representaremos respectivamente con los términos: Gobierno, Unidades de

\footnotetext{
${ }^{3}$ Vale la pena considerar el concepto de stakeholders, de origen inglés acuñado por Freeman en su obra Strategic Management: A Stakeholder Approach (PITMAN, 1984), el cual se refiere a "quienes pueden afectar o son afectados por las actividades de una empresa". Dichos grupos son los interesados que, de acuerdo con Freeman, deben ser considerados como un elemento esencial en la planificación estratégica de los negocios. Por tanto, la innovación, como creación de valor económico y social, debería implicar que todos los stakeholders reciban, en lo posible, algún beneficio o al menos que ninguno de ellos reciba un perjuicio.
} 
Investigación, Empresas y Sociedad, a partir de los cuales se construye el nombre del sistema propuesto: GUIES. Las nuevas teorías regionales destacan la importancia del territorio, como soporte para la competitividad empresarial y como entramado de instituciones públicas y privadas para gestionar el desarrollo (MADOREY, 1999), representando concepciones integrales y dinámicas del proceso de desarrollo. Esas dinámicas se sustentan especialmente en los componentes intangibles del territorio: su nivel de organización, la capacidad de gestión de sus instituciones, la capacidad emprendedora, el aprendizaje, la actitud de cooperación y cambio; aspectos que se constituyen en los principales acervos que favorecen las transformaciones de los sistemas productivos y su inserción competitiva.

\section{Conceptos y premisas básicas del modelo}

\subsection{Desarrollo territorial: el "para qué" de un SRIDT}

El concepto de desarrollo territorial expresa en un determinado grupo poblacional su dinamismo económico, la distribución del ingreso, dotación y calidad medio ambiental, satisfacción de las necesidades básicas y de las condiciones de libertad, felicitad y realización personal (JIMÉNEZ, 2001).

De acuerdo con Vázquez-Barquero (1988) el desarrollo local se define como

un proceso de crecimiento económico y de cambio estructural que conduce a una mejora en el nivel de vida de la población local, en el que se pueden identificar tres dimensiones: una económica, en la que los empresarios locales usan su capacidad para organizar los factores productivos locales con niveles de productividad suficientes para ser competitivos en los mercados; otra, sociocultural, en que los valores y las instituciones sirven de base al proceso de desarrollo; y, finalmente, una dimensión político-administrativa en que las políticas territoriales permiten crear un entorno económico local favorable, protegerlo de interferencias externas e impulsar el desarrollo local.

[...] el ritmo de cambio tecnológico y mundialización requiere rápidos cambios estructurales tanto en los mercados de los países en desarrollo como en los de los desarrollados [...] Así como la Gran Depresión se debió en parte a las dificultades para pasar de una economía agraria y rural a otra urbana y manufacturera, así también los problemas actuales se deben en parte a la necesidad de pasar de la manufactura a los servicios. Se deben crear nuevas empresas, pero los mercados financieros modernos son mejores para la especulación y la explotación que para aportar fondos para nuevas empresas, en particular las pequeñas y las medianas. Además, para hacer la transición hacen falta inversiones en capital humano que con 
frecuencia las personas no pueden costear. Entre los servicios que las personas necesitan figuran la salud y la educación, sectores en los que el Estado desempeña de forma natural un papel importante (dadas las imperfecciones inherentes a los mercados en esos sectores y las preocupaciones por la equidad). (STIGLITZ, 2013)

Tanto el avance y desarrollo tecnológico, como los avances en innovación deben estar orientados para lograr un avance económico, bajo la premisa que la población tenga avances en salud, educación, conocimiento, respeto por el medio ecológico, respeto por las diferentes culturas y diversas formas sociales que se puedan establecer, esto quiere decir, que se establezca un desarrollo integral.

Piketty (2014) da testimonio de la cada vez mayor preocupación sobre la creciente desigualdad. El desarrollo territorial, debe estar fundamentado para alcanzar la equidad y la igualdad social, en términos de llegar a ofrecer y garantizar los derechos mínimos sociales, especialmente en salud y educación; con indicadores de desarrollo complementarios al PIB, que contemplen la desigualdad, medida a través del índice de GINI y como la inequidad que puede ser medida a través de la falta de acceso a la salud o a la educación. Hablar de inequidad, implica hablar de equidad y para ello, se hace necesario enfatizar dos principios que subyacen al análisis de la misma: el principio de equidad horizontal: situaciones similares debieran recibir un tratamiento similar, y el principio de equidad vertical: situaciones diferentes merecen ser tratadas de forma diferente (STARFIELD, 2001). Es claro que en Colombia con un incremento en el PIB per/cápita no se ha evidenciado un avance en términos de igualdad y equidad.

El desarrollo territorial evoluciona, por lo tanto no se puede diseñar un SRIDT genérico. Cada región tiene, en su momento, connotaciones particulares y no, necesariamente, iguales a las de otras regiones. Estas particularidades también evolucionan con el tiempo y, por tanto, el SRIDT debe ser revisado y ajustado periódica y sistemáticamente, acorde con los procesos evolutivos y de esta forma, concertarlo a los nuevos impactos que deberían logarse en función del bienestar territorial. Además, la conceptualización y apropiación del término bienestar territorial debe ser construida conjuntamente con los beneficiarios del territorio bajo unas claras dimensiones: perspectiva de inclusión, integralidad y apropiación social del conocimiento; a partir de lo cual, los proyectos de innovación deberían contemplar, dentro de sus objetivos, la creación de valor tanto económico como social. 


\subsection{Innovación: el "cómo crear valor económico y social para el DT"}

En una sociedad como la actual, donde los cambios se suceden ininterrumpidamente, la innovación y el conocimiento se convierten en los principales motores de la economía (VASCONCELOS et al., 2008).

El concepto de innovación asumido es el presentado por el Departamento Nacional de Planeación de Colombia en el lanzamiento de la Estrategia Nacional de Innovación: "Innovación es la asimilación y explotación exitosa de una novedad, en las esferas económica y social, de forma que aporte soluciones nuevas a los problemas nacionales y regionales y permita así responder a las necesidades de la sociedad y el sector productivo" (DNP, 2011).

Cabe recalcar la importancia de incluir, además de las innovaciones tecnológicas, todo tipo de innovaciones que conduzcan a la creación de valor económico y social, en función del incremento de la productividad, incluyendo el mejoramiento continuo, mediante la aplicación de conocimiento a las tareas rutinarias, válido hasta que dicho incremento de productividad se sature en virtud de la ley de los rendimientos decrecientes, lo que determina pasar a la innovación, mediante la aplicación de conocimiento a nuevas tareas, conduciendo al logro de incrementos sustanciales en la productividad.

Consecuentemente, los proyectos de innovación deben estar orientados al incremento de la productividad, contemplando en sus objetivos, tanto la creación de valor económico como de valor social, en función del desarrollo territorial.

Es por ello que para significar la tradicional $\mathrm{I}+\mathrm{D}+\mathrm{I}$ hablamos de $\mathrm{I}+\mathrm{D}+\mathrm{D}+\mathrm{I}$ (PÉREZ-CASTAÑO, 2011), esto es, identificación de oportunidades para crear valor social y económico (lo cual requiere de la investigación), desarrollo de ideas, desarrollo de proyectos e impacto (en términos de beneficios para los stakeholders y tanto de productividad como de competitividad para las empresas); destacando que el detonador debería ser la identificación de oportunidades para obtener beneficios en función del desarrollo territorial y diferenciamos el desarrollo de ideas y el desarrollo de proyectos porque son de diferente naturaleza y la gestión de cada uno tiene sus propias particularidades. 


\subsection{Sistema: el "cómo lograr las interacciones entre los agentes del SRI para contribuir al DT"}

Un concepto generalizado de sistema es un conjunto de elementos coordinados entre si y estructurados deliberadamente para lograr propósitos. En nuestro caso, los elementos son los actores regionales Gobierno, Unidades de Investigación, Empresas y Sociedad y por ello, el nombre del sistema propuesto es GUIES. Estos actores deben estar coordinados y estructurados deliberadamente, lo que implica establecer una estructura organizacional con una dirección que actúe de manera similar a la dirección de una orquesta sinfónica, conociendo y comprendiendo perfectamente los acordes de cada conjunto de instrumentos, cuya principal función es armonizar dichos acordes.

Por tanto, la dirección del SRI debe conocer y comprender en profundidad las necesidades prioritarias de la sociedad, la región, la función pública, la idiosincrasia y las particularidades del oficio de la red empresarial, como de los investigadores y de esta forma se puedan potenciar sus diversas fortalezas; debe tener la habilidad de armonizar y consensuar las discrepancias para integrar la diversidad de intereses de los distintos actores, en pro del desarrollo territorial.

\section{Sistema Universidad - Empresa - Estado}

A continuación se presenta una breve revisión de la literatura sobre los sistemas nacionales de innovación y su relación con el sistema universidad, empresa, estado, como sistemas de conocimiento.

Los primeros ensayos sistemáticos basados en la teoría acerca de sistemas nacionales de innovación fueron de List (1885), destacado economista alemán y considerado el teórico original de la unión europea; List hace una distinción entre la aproximación cosmopolita de Adam Smith, la cual se centra en la localización y el cambio y su perspectiva nacional para el desarrollo de fuerzas productivas. El elemento de List es completo, complejo y rico; en su análisis destacando la necesidad de que el gobierno asuma su responsabilidad en la educación y capacitación y en el desarrollo de una infraestructura que soporte el desarrollo industrial, además como un argumento para proteger a las pequeñas industrias.

El primer uso explícito del concepto de sistema nacional de innovación, lo trata Freeman (1987), con el cual se refiere a la organización de subsistemas específicos en 
una nación y a la interacción entre estos subsistemas. Freeman se centra en la interacción entre los sistemas de producción y los procesos de innovación; aplica una combinación de la organización y la teoría de la innovación: cuáles formas organizacionales conducen más al desarrollo y al uso eficiente de nueva tecnología? La organización de la I+D y de la producción en las empresas, las relaciones entre las empresas y el papel del gobierno son el centro de su análisis, a partir de lo estudiado en Japón

Nelson (1974) presentó estudios de los sistemas americanos de innovación siendo el centro del análisis la combinación del carácter público y privado de la tecnología y el papel respectivo de las empresas, del gobierno y de las universidades en la producción de nueva tecnología, mostrando que los diferentes sectores industriales usan diversos métodos para apropiar los resultados de sus innovaciones. El núcleo en el trabajo de Nelson es la producción de conocimiento e innovación en sentido reducido, estando las herramientas teóricas relacionadas con leyes y economías: cómo pueden las diferencias institucionales establecer y solucionar el dilema público y privado de la información y la innovación técnica?

Porter (1990) muestra cuatro factores distintos determinantes que afectan la competitividad de una industria nacional: Estrategia de la empresa, Factores condicionantes, Condiciones de la demanda y Soporte de las industrias, lo cual puede verse como un trabajo en los sistemas nacionales de innovación.

Los avances científicos y tecnológicos han tenido un impacto considerable en los procesos socio-económicos por el cambio debido a las innovaciones tecnológicas (LEYDESDORFF; VAN DEN BESSELAAR, 1994); consecuentemente, las condiciones socioeconómicas juegan un papel importante en los procesos de toma de decisiones relacionado con $\mathrm{I}+\mathrm{D}$, basados en los sistemas de conocimiento, especialmente en el sector industrial; en este sentido, Parikh (2001) enfatiza que un objetivo primordial de un sistema de conocimiento es apoyar la transformación del conocimiento adecuadamente para su distribución e intercambio entre las partes interesadas.

Pol y Carroll (2006) han argumentado que el sistema de conocimiento favorece de manera fundamental el cambio económico, en el cual las componentes de innovación, actividades de emprendimiento y el poder de mercado, juegan un papel 
importante. Comparativamente, Drucker (1985) considera la innovación como "un instrumento específico del emprendedor" y un "resultado de los sistemas basados en el conocimiento". Para Lindley (2003), un sistema de conocimiento, al igual que una sociedad, es "un proceso de cambio estructural que conduce a la difusión de la producción y uso del conocimiento en la economía con un potencial para desempeñar un papel importante en la creación de riqueza”. Twarog (2003) describe los sistemas de conocimiento como entidades compuestas de sistemas de investigación, instituciones de educación superior, las industrias y los gobiernos, los órganos de formulación de políticas, y laboratorios de $\mathrm{I}+\mathrm{D}$, que integran varios factores de innovación y sus respectivos mecanismos de ayuda.

Leydesdorff y Meyer (2006) se refieren a un sistema de innovación basada en el conocimiento como: "un resultado de la interacción entre los diferentes mecanismos de coordinación social, como los mercados, la producción de conocimiento y la gobernanza de las interfaces". Edquist (1997) señala que un sistema de conocimiento puede permanecer activo en los diferentes niveles (por ejemplo, industrial, local, regional, nacional, e internacional). Según Etzkowitz y Leydesdorff (1995), los modelos de sistemas de conocimiento muestran el flujo, la reorganización y la expansión del papel del conocimiento en la sociedad y la economía.

\section{Sistema (GUIES) Gobierno - Unidades de Investigación - Empresas - Sociedad}

En esencia se debe buscar que la inversión en I+D que realizan los gobiernos sea más eficaz y eficiente en términos del incremento de la productividad, de la competitividad y del desarrollo territorial, se trata de la búsqueda del equilibrio entre competitividad, sostenibilidad y cohesión social.

\subsection{Elementos del Sistema GUIES}

Para mejorar la eficiencia y la eficacia de la inversión en I+D, la frecuencia y la intensidad de las relaciones entre las unidades de investigación y las empresas deberían ser permanentes, dinámicas y sistemáticas, lo cual se puede lograr conformando un sistema, de tal manera que, satisfaciendo las necesidades del mercado, procure el bienestar de la sociedad, contribuyendo al desarrollo territorial.

La Figura 1 muestra el esquema de este sistema destacando que, tanto la investigación en las unidades de investigación como la producción en las empresas, si 
bien es cierto deben buscar la satisfacción de necesidades del mercado, deben sobretodo tener en cuenta el impacto en el desarrollo de la sociedad, a su vez, la sociedad le debe plantear, tanto a los unidades de investigación como a las empresas, nuevas necesidades para su desarrollo; esto es, debe haber una interacción de tipo sistémico entre sociedad, empresas y unidades de investigación, y, consecuentemente, entre desarrollo, producción e investigación.

Por tanto, la investigación (el saber) debe orientarse para apoyar la producción (el hacer) de bienes, productos y servicios, que impacten positivamente el desarrollo de la sociedad. Le competen al gobierno varias funciones en este sistema, entre ellas: propiciar, fomentar y financiar relaciones eficaces entre sociedad, empresas y unidades.

De otra parte, la interacción entre unidades y empresas no solo debe buscar la manera de resolver problemas para incrementar la productividad en los procesos y productos existentes, sino con procesos y productos diferentes, mediante la planeación y ejecución conjunta de proyectos conducentes al diseño y desarrollo de técnicas, modelos, materiales, productos que den respuesta a necesidades existentes en el mercado o que permitan aprovechar oportunidades potenciales, que impacten positivamente el desarrollo de la sociedad.

Figura 1 - Sistema GUIES

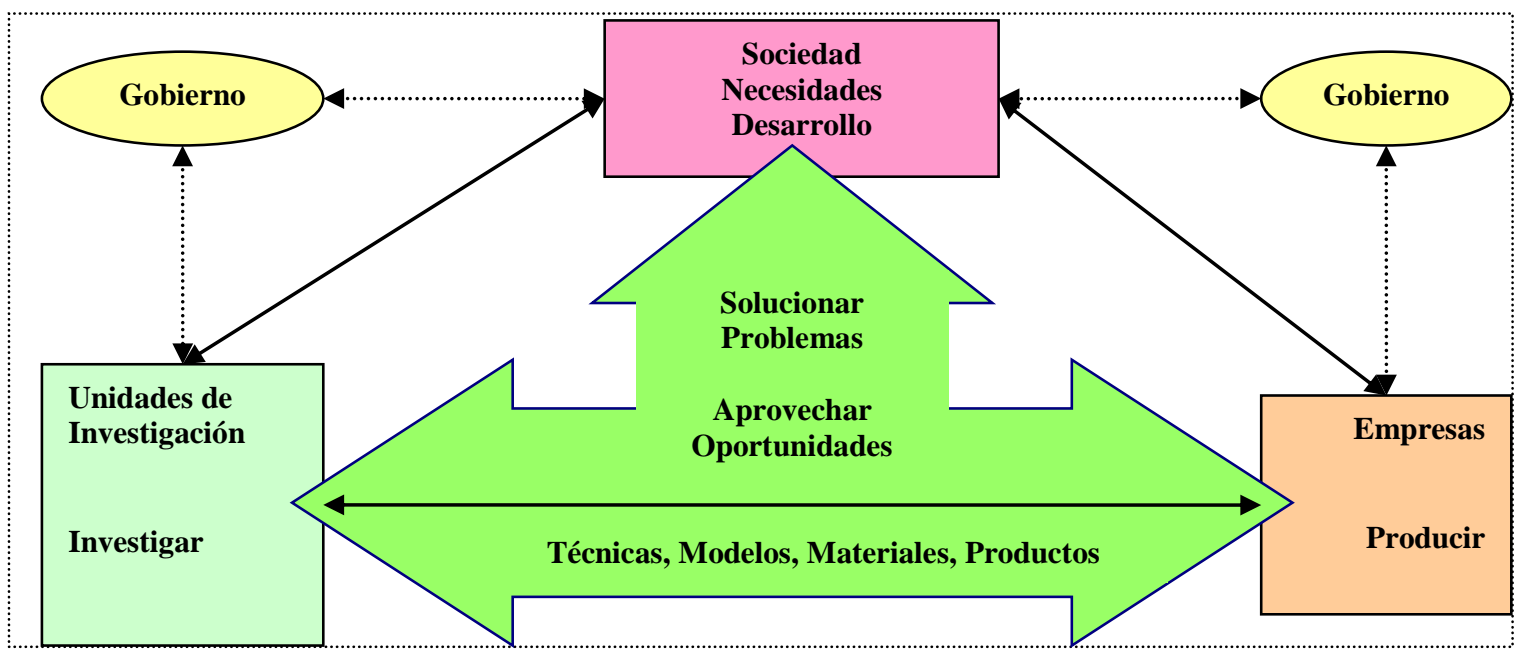

Fuente: Pérez-Castaño (2006).

\subsection{Operación del Sistema GUIES}

En la Figura 2 se representa el Sistema GUIES a la manera tradicional de un sistema de producción, con entradas, procesos, salidas y su respectiva retroalimentación. En este caso parte fundamental de las entradas está constituido por las capacidades del 
sistema representadas en conocimientos, habilidades y destrezas del personal de la unidades de investigación, de las empresas, del gobierno y en general de la sociedad, jugando un papel crucial el conocimiento específico que se tenga sobre los resultados y el impacto previstos para el Sistema GUIES; con base en las capacidades mencionadas se procesan los recursos, de manera integral, a través de las diferentes etapas de investigación, desarrollo y producción buscando resultados acordes con lo previsto, en términos de agregar valor, representado en productividad, innovación y capital intelectual; resultados cuyo último fin es impactar en el desarrollo de las instituciones, de la región y la sociedad. La retroalimentación está conformada esencialmente por la gestión de conocimiento orientada a la agregación de valor en todo el sistema, mediante los procesos relacionados con la identificación y adquisición de los conocimientos requeridos; difundir y compartir dichos conocimientos; asimilar y aplicarlos debidamente; para finalmente dar paso a la creación de nuevos conocimientos. Se trata entonces de un Sistema I (Identificación de oportunidades para crear valor social y económico, lo cual requiere de la investigación)+D (desarrollo de ideas) + D (desarrollo de proyectos)+I (Impacto), en el cual el fin último lo constituye el impacto causado por la innovación.

Figura 2 - Operación del Sistema GUIES

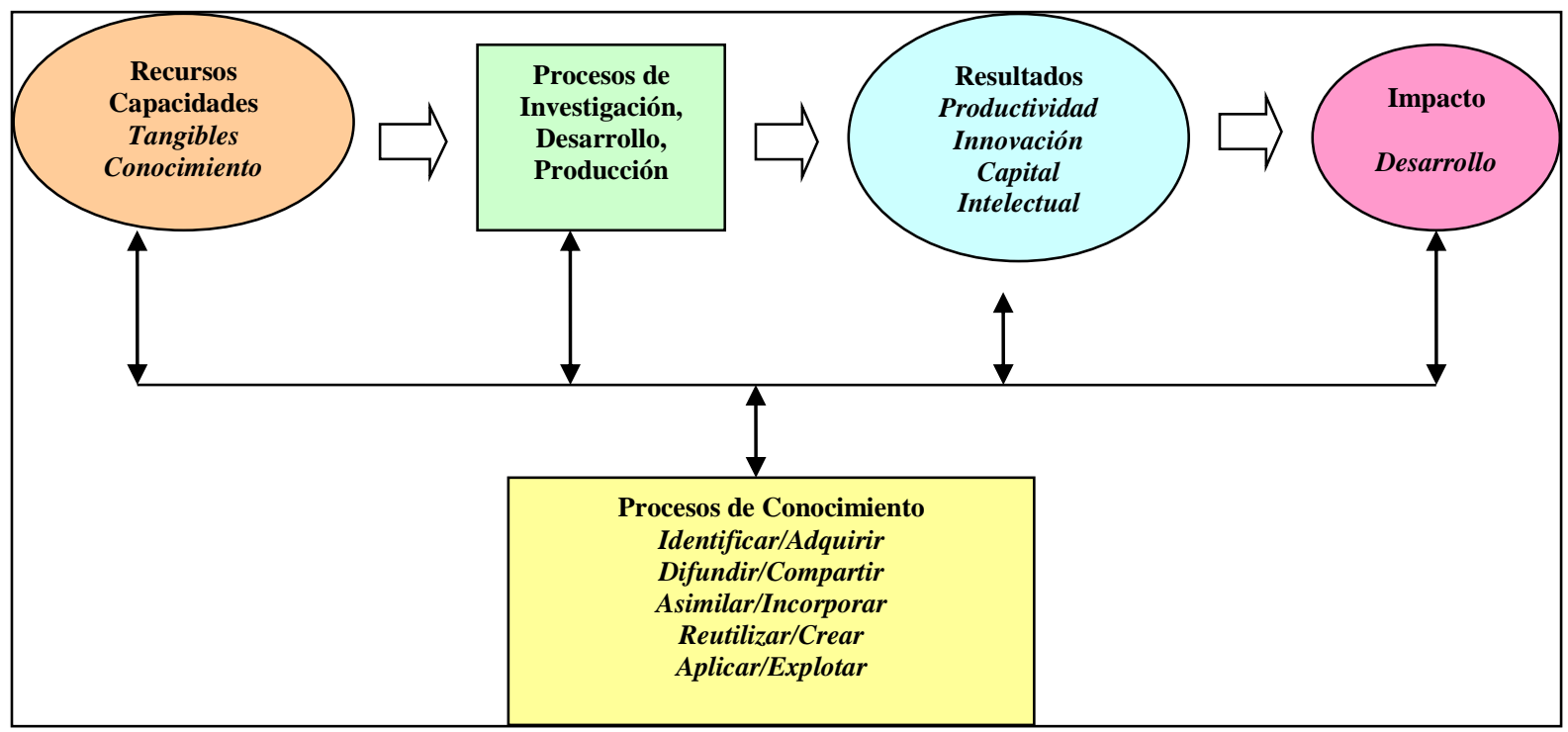

Fuente: adaptado de Pérez-Castaño (2001), Bueno (2003) e Dalmau, Pérez e Baixauli (2005).

\subsection{Capital Intelectual del Sistema GUIES}

En la Figura 3 se representa el balance del capital intelectual del Sistema GUIES. El gobierno, el sistema científico tecnológico y el sistema productivo aportan 
recursos y capacidades ( $\mathrm{R} \& \mathrm{C})$ a cada proyecto, logrando unos resultados en términos de incremento en el capital intelectual de cada uno de los aportantes y de la región, causando a su vez un desarrollo, tanto para la sociedad, en general, como para cada uno de los subsistemas que integran el Sistema GUIES.

Figura 3 - Capital Intelectual del Sistema GUIES

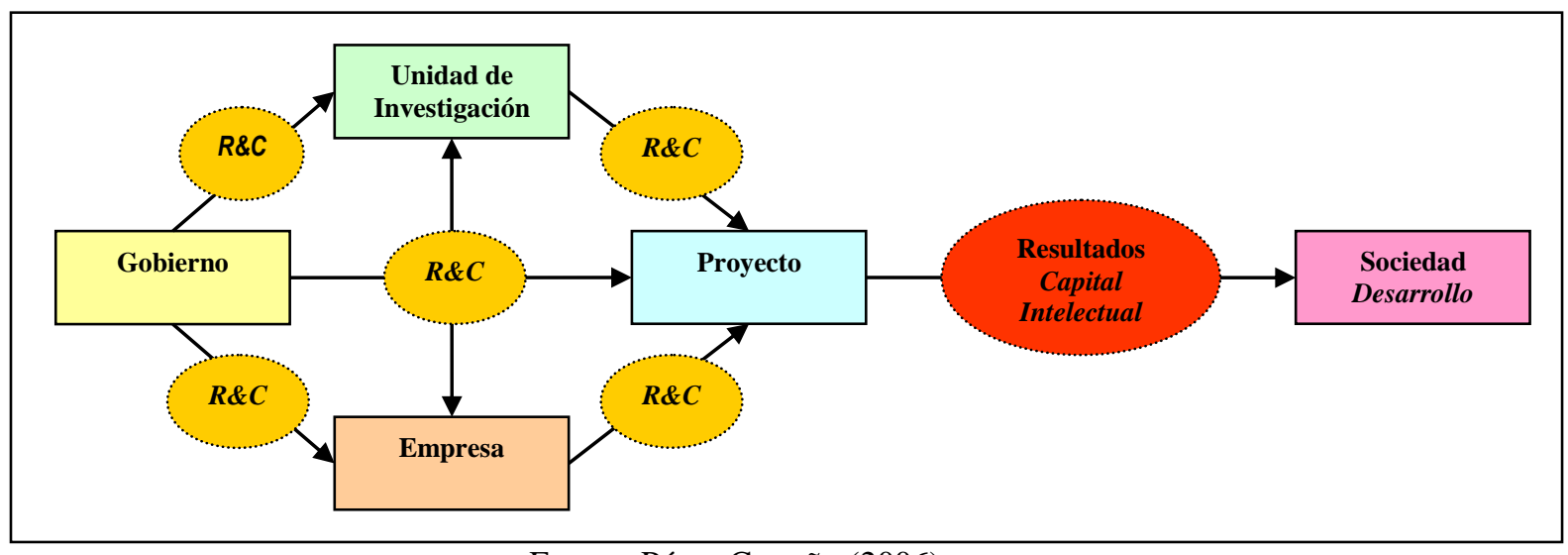

Fuente: Pérez-Castaño (2006).

\subsection{Gestión del Sistema GUIES}

En la Tabla 1 se relacionan los elementos necesarios para construir el cuadro de mando integral que permite planificar y gestionar el Sistema GUIES. El punto de partida es la visión y los objetivos estratégicos de la región y de cada uno de los subsistemas, representados mediante indicadores de productividad, innovación y creación de nuevas organizaciones, con base en lo cual se deben identificar los resultados esperados en términos de los diferentes componentes del capital intelectual (humano, organizacional, relacional y tecnológico); para posteriormente identificar las actividades clave que se deben desarrollar, los recursos y capacidades necesarios, para finalmente definir los proyectos, programas y planes que se deben formular. 
Tabla 1 - Cuadro de Mando Integral para la Planificación y Gestión del Sistema GUIES

\begin{tabular}{c|c|c|c|c|c} 
VISIÓN & CAPITAL & RESULTAD & ACTIVIDADE & RECURSOS & PLANES \\
OBJETIVOS & INTELECTU & OS & S & CAPACIDAD & PROGRAM \\
ESTRATÉGIC & AL & DESEADOS & A & ES & AS \\
OS & & & $\begin{array}{c}\text { DESARROLL } \\
\text { AR }\end{array}$ & $\begin{array}{c}\text { NECESARIO } \\
\text { S }\end{array}$ & $\begin{array}{c}\text { PROYECT } \\
\text { OS }\end{array}$ \\
\hline $\begin{array}{c}\text { Productividad } \\
\text { Innovación } \\
\text { Nuevas } \\
\text { Empresas }\end{array}$ & Capital & Humano & & & \\
\hline $\begin{array}{c}\text { Productividad } \\
\text { Innovación } \\
\text { Nuevas } \\
\text { Empresas }\end{array}$ & $\begin{array}{c}\text { Capital } \\
\text { Organizacional }\end{array}$ & & & & \\
\hline $\begin{array}{c}\text { Productividad } \\
\text { Innovación } \\
\text { Nuevas } \\
\text { Empresas }\end{array}$ & $\begin{array}{c}\text { Capital } \\
\text { Relacional }\end{array}$ & & & & \\
\hline $\begin{array}{c}\text { Productividad } \\
\text { Innovación } \\
\text { Nuevas } \\
\text { Empresas }\end{array}$ & $\begin{array}{c}\text { Capital } \\
\text { Tecnológico }\end{array}$ & & & & \\
\hline
\end{tabular}

Fuente: adaptado de Dalmau, Pérez y Baixauli (2005).

\subsection{Gestión de conocimiento para la creación de valor económico y social}

Una empresa podría lograr el éxito por uno de los tres caminos, representados por las ideas, las capacidades y las conexiones; consecuentemente, una región puede prosperar como centro de categoría internacional de generadores de ideas (pensadores), transformadores de ideas en productos y servicios (fabricantes) o vendedores de productos y servicios (comerciales).

Sin embargo hay un elemento adicional e importante que no se encuentra explícito en este modelo, que tradicionalmente se viene utilizando para la gestión de la innovación, el impacto social que debe tener la innovación en términos de desarrollo territorial.

En la Figura 4 se representa la gestión de conocimiento para la creación de valor económico y social con el beneficio para el desarrollo territorial. 
Figura 4 - Gestión de conocimiento para la creación de valor económico y social

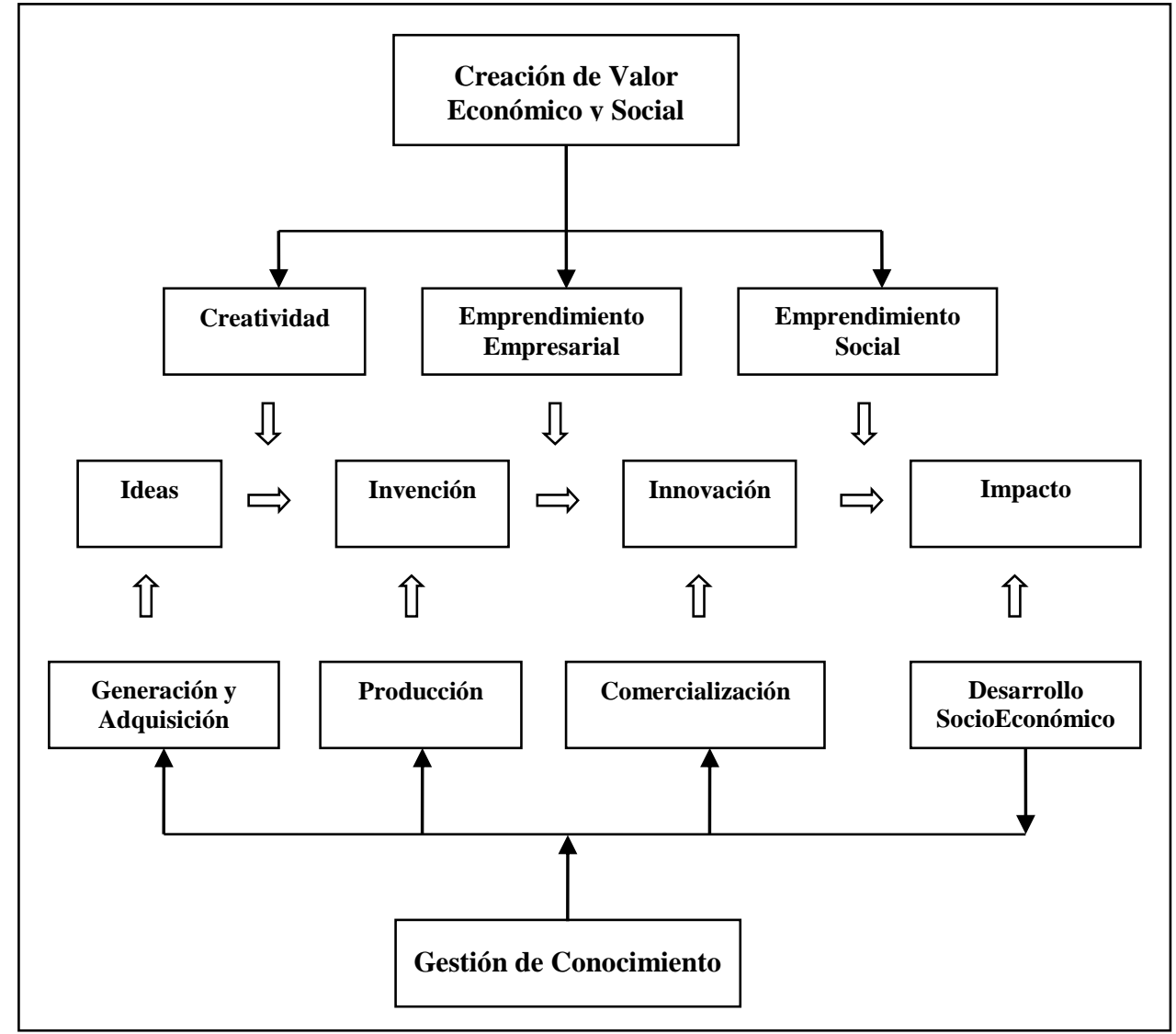

Fuente: Pérez-Castaño (2006).

Las ideas (implican actividades de adquisición y generación de conocimientos), acompañadas de creatividad, se materializan en invenciones (implican actividades de producción de bienes y servicios); las invenciones, a su vez, acompañadas de emprendimiento empresarial se transforman en innovaciones (implican actividades de comercialización); innovaciones que acompañadas de emprendimiento social (implican actividades para satisfacer necesidades asociadas con el desarrollo territorial) causan un impacto socio económico en la región.

A continuación se describe cada grupo de actividades:

- $\quad$ Actividades de generación y adquisición de conocimiento.

La empresa tiene dos alternativas para generar o adquirir el conocimiento, tanto de tipo hardware como software, necesario para llevar a cabo la innovación: internamente mediante la investigación y desarrollo (I+D) o adquirirlo del exterior.

- $\quad$ Actividades para la producción o provisión de servicios 
Están relacionadas directamente con el proceso de transformaciones del conocimiento y tecnología adquiridas en mejoras para la empresa, tanto de producto o servicio como de proceso. Las tres actividades básicas que integran esta fase del proceso son: El diseño industrial e ingeniería de producto, la ingeniería de proceso y el lanzamiento de la fabricación.

- $\quad$ Actividades para la comercialización y utilización.

Se deben realizar los estudios necesarios para determinar el impacto y la compatibilidad de la innovación con los consumidores, el mercado y en general la sociedad.

\section{- $\quad$ Actividades para el desarrollo socio económico}

Se deben realizar los estudios necesarios para determinar en qué medida la innovación contribuye al logro de los objetivos estratégicos de la región, en términos de su desarrollo socio económico.

\section{Consideraciones finales}

Frente a un cúmulo de oportunidades, los nuevos desafíos que enfrentan los ecosistemas de innovación regionales en Colombia son graves, y serán insuperables, a menos que se inicien acciones correctivas serias. Pero no se puede generalizar, es importante tener presente que el papel que desempeñan los sistemas de conocimiento de las instituciones de educación superior, de las empresas y del gobierno, difieren sustancialmente de una región a otra, en función de varias dimensiones, entre ellas, de posicionamiento socio-económico, cultural, demográfica, geográfica, política, con una compleja mezcla de problemas diversos y diferentes, algunas regiones carecen de las necesidades básicas de alimentación, salud, educación, agua potable, mientras que, en contraste, otras cuentan con capacidades superiores que les permiten avanzar más fluidamente en el campo tecnológico.

Se requiere profundizar en la identificación clara de las barreras a la innovación para salvar la brecha existente en cuanto a colaboración, producto de la falta de interacción entre los agentes de innovación, clave en un ecosistema de la innovación. Para ello se recomienda examinar cuidadosamente el papel de los sistemas de conocimiento propios y sui generis de cada uno de los actores. 
El gobierno colombiano, los departamentales y los locales, deberían promover decididamente la innovación conducente a la creación de valor económico y social, para hacer frente al reto de crear empleo y riqueza, tal como lo hacen los gobiernos en las economías modernas, en virtud de la evidencia de las contribuciones de la innovación, percibidas para la creación de empleo y riqueza, de acuerdo con Orhan y Scott, (2001).

Las universidades deberían orientar con mayor eficacia su papel en la formación de emprendedores para ser vistas como sistemas de innovación de la sociedad, jugando un papel destacado en el desarrollo socio-económico, dado que su tarea no es sólo formar individuos orientados y competentes empresarialmente, sino también fomentar los mecanismos sociales que sostienen y facilitan el surgimiento de nuevas empresas y el crecimiento de las existentes a nivel regional, tal como lo anota Laukkanen (2000).

La eficiencia del sistema de innovación y su rendimiento depende de las actividades del conocimiento, ya que los sistemas de conocimiento están estrechamente relacionados con la innovación y el desarrollo socio-económico a través de la interconexión para la introducción de conocimiento en la economía y la sociedad en general, mediante un flujo eficiente de conocimiento por la interacción de los actores regionales, tal como lo anotan Lundvall et al. (2002), Reynolds et al. (2002), Edquist et al. (2000), Parikh (2001), Carree y Thurik (2002); lo cual, una vez más ratifica la necesidad de gestionar el conocimiento de manera integral y holística en las regiones con la amplia participación de todos y cada uno de los agentes que participan en las innovaciones

Sólo mediante la promoción de una interacción, con la premisa de ganar-ganar, entre el gobierno, la industria y las instituciones de educación superior, pueden la innovación y el emprendimiento proliferar en beneficio del ecosistema de innovación, conducente a la creación de valor económico y social para el desarrollo territorial. La promoción de la innovación depende de las funciones desarrolladas por los diversos grupos de interés, actores importantes en el sistema de conocimiento, para poder integrar, eficaz y eficientemente, los nuevos conocimientos en la innovación. De ahí la importancia de comprender las funciones desempeñadas por las partes interesadas clave, para conciliar, consensuar e integrarlas en función del desarrollo territorial.

El desarrollo territorial evoluciona, por lo tanto no se puede diseñar un SRIDT genérico. Cada región tiene, en su momento, connotaciones particulares y, no 
necesariamente, iguales a las de otras regiones. Estas particularidades también evolucionan con el tiempo y, por tanto, el SRIDT debe ser revisado y ajustado periódica y sistemáticamente, acorde con los procesos evolutivos y de esta forma, concertarlo a los nuevos impactos que deberían logarse en función del bienestar territorial. Consecuentemente, el Plan de Desarrollo Regional, el Plan Regional de Competitividad e Innovación y el Plan de CTeI se deberían realizar de manera integral y simultánea. En definitiva, es por estas razones que tanto los planes de desarrollo, de CTeI, como los planes de competitividad, deben revisarse periódicamente de acuerdo a las nuevas necesidades detectadas y así lograr el bienestar deseado. la actualización periódica del inventario de capacidades, recursos y necesidades de la región.

De otra parte, en función de los recursos, capacidades, necesidades y vocación de la región, se deben priorizar sectores o actividades tanto industriales como de otra índole y, a su vez, elegir en cada uno de ellos o en todos, si la región va a enfatizar ser: un proveedor de insumos, un procesador de insumos, un proveedor de productos terminados o un consumidor.

Consecuentemente, el plan estratégico de innovación de una región se debe centrar en cadenas productivas estratégicas que, con base en los conocimientos, la tecnología y la innovación, puedan elevar en el tiempo el nivel de valor agregado de lo producido, a partir de la apropiación socio productiva del conocimiento, y conducir a las empresas hacia una organización basada en el conocimiento y la innovación, que permita el desarrollo de las propias capacidades de las personas y la generación de desarrollo humano y sostenibilidad. Para ello, el incremento en el valor agregado debe reflejar un ingreso económico justo y proporcional, tanto al esfuerzo realizado como a la contribución en el valor creado en cada una de los eslabones de la cadena productiva, de tal manera que impacte positivamente, tanto el PIB per cápita como el Índice Gini del territorio.

Gran número de diagnósticos coinciden en que el problema central de la CTeI en Colombia radica en deficiencias para adquirir, producir, aplicar e integrar conocimiento, lo cual está estrechamente relacionado con el aprendizaje, de ahí la importancia de que las políticas industriales identifiquen sectores industriales donde el aprendizaje pueda generar beneficios en la economía. 
Las políticas industriales - mediante las cuales los gobiernos intervienen en la asignación de recursos entre sectores o intervienen para favorecer más a algunas tecnologías en comparación con otras - pueden ayudar a aprender a las "economías incipientes". El aprendizaje puede ser más marcado en algunos sectores que en otros, y los beneficios de dicho aprendizaje, incluyendo el desarrollo institucional necesario para el éxito, podría extenderse a otras actividades económicas.

Del mismo modo, la propiedad intelectual, si no se la diseña correctamente, puede ser un arma de doble filo cuando se la ve desde una perspectiva de aprendizaje. Si bien puede aumentar los incentivos para invertir en investigación, también puede aumentar los incentivos para actuar con secretismo, lo que impide el flujo de conocimiento, esencial para el aprendizaje, mientras que al mismo tiempo alienta a que las empresas maximicen lo que obtienen del conjunto de conocimientos colectivos y minimicen lo que aportan a dicho conjunto. En este escenario, el ritmo de innovación, en los hechos, se reduce.

Prácticamente cada una de las políticas gubernamentales, de forma deliberada o no, para bien o para mal, tiene efectos directos e indirectos sobre el aprendizaje. Los países en desarrollo, donde los formuladores de políticas están conscientes de estos efectos, son más propensos a cerrar la brecha de conocimiento que los separa de los países más desarrollados

Hace un siglo, el economista y politólogo Joseph Schumpeter argumentó que la virtud central de la economía de mercado era su capacidad para innovar. Sostuvo que el enfoque tradicional de los economistas sobre los mercados competitivos se ubicaba en el lugar equivocado; lo que importaba era la competencia por el mercado, no dentro del mercado. La competencia por el mercado fue lo que condujo a la innovación. Una sucesión de monopolistas conduciría, según ese punto de vista, a niveles de vida superiores en el largo plazo.

Si bien una parte del incremento de la productividad refleja el impacto que tienen los grandes y espectaculares descubrimientos, una gran parte se ha debido a cambios pequeños y graduales. Y, si ese es el caso, tiene sentido centrar la atención en cómo las sociedades aprenden y qué es lo que se puede hacer para promover el aprendizaje, incluyendo cómo aprender a aprender. 
Se deben aprovechar al máximo las ventajas comparativas de las empresas y en las oportunidades que le brinda el entorno, utilizándolas estratégicamente para adquirir ventajas competitivas, orientándose hacia la incorporación de conocimiento y tecnología en sus recursos y capacidades existentes y potenciales, buscando obtener un incremento sostenido en el valor agregado, consecuentemente en los ingresos, pero sobretodo buscando un mejor nivel y calidad de vida para los habitantes de la región y en general para los colombianos.

Incentivar el desarrollo de proyectos de investigación de acuerdo con el impacto según los indicadores escogidos como claves para el DT. Frederick Terman, trabajó como rector, profesor y decano de ingeniería de la Universidad de Stanford, considerado como el "padre de Silicon Valley", convirtió la escuela de ingeniería en ciernes de Stanford en un motor de innovación, alentando a los departamentos de ciencias e ingeniería para que trabajaran juntos, vinculándolos a empresas locales, y centrando la investigación en las necesidades de la industria; creó una cultura de cooperación e intercambio de información que desde entonces ha definido la región. Las altas tasas de cambios de empleo y formación empresarial de Silicon Valley, sus redes profesionales y la facilidad de intercambio de información, fue lo que le dio ventaja. La empresas de Valley entendieron que colaborar y competir a la vez conducía al éxito, una idea que incluso se refleja en la inusual regla californiana de prohibir los acuerdos de no competencia. El ecosistema daba apoyo a la experimentación, a la toma de riesgos y al intercambio de lecciones de éxito y fracaso. En otras palabras, Silicon Valley era un sistema abierto, una red social gigante del mundo real que existía mucho antes que Facebook.

\section{AGRADECIMIENTOS}

Los autores manifiestan su agradecimiento por el apoyo obtenido de la Universidad Politécnica de Valencia de manera directa y por su intermedio del Ministerio de Ciencia e Innovación de España para desarrollar las bases de este modelo en estancias posdoctorales.

\section{REFERENCIAS}

BASSANINI, A.; SCARPETA, S.; VISCO, I. Knowledge, Technology and Economic

Growth: Recent Evidence from OECD-countries. París: OCDE, 2000. 
BOISIER, S. El vuelo de una cometa. Una metáfora para una teoría del desarrollo territorial. ILPES - Instituto Latinoamericano y del Caribe de Planificación Económica y Social en Teoría y metáforas sobre el desarrollo territorial, 1997. Disponible en: $<$ http://www.cepal.org/cgibin/getProd.asp?xml=/publicaciones/xml/5/5035/P5035.xml\&xsl=/tpl/p9f.xsl\&base=/tp 1/top-bottom.xsl>. Acceso en: 20 mai. 2015.

CAMPOS, E. B. Gestión del Conocimiento en Universidades y Organismos

Públicos de Investigación. Edita Dirección General de Investigación, Consejería de Educación Comunidad de Madrid, 2003.

CARREE, M.; THURIK, A. R. The Impact of Entrepreneurship on Economic Growth. In: ACS, Z.; AUDRETSCH, D. B. International Handbook of Entrepreneurship Research. Boston/Dordrecht: Kluwer Academic Publishers, 2002.

\section{DALMAU, J. I.; PÉREZ-CASTAÑO, B. J.; BAIXAULI, J. J. Technology Transfer} between Research Units and Enterprises. An approach to centered model in the Impact on Territorial Strategic Targets. Papers on Territorial Intelligence and Governance Participative Action-Research and Territorial Development. International Conference of Territorial Intelligence. Huelva: Observatorio Local de Empleo, 2007. DNP. Lanzamiento de la Estrategia Nacional de Innovación (ENI), 8 de septiembre de 2011.

DOSI, G.; FREEMAN, C.; NELSON, R.; SILVERBERG, G.; SOETE, L. Technical change and economic theory. London, N.Y.: Pinter Publishers, 1988.

DRUCKER, P. Innovation and Entrepreneurship Practice and Principles. New York: Harper \& Row, 1985.

EDQUIST, C. (Ed.) Systems of Innovation: Technologies, Institutions and Organizations. London: Pinter/Cassell, 1997.

EDQUIST, C.; ERICSSON, M-L.; SJÖGREN, H. Collaboration in Product Innovation in the East Gothia Regional System of Innovation. Enterprise \& Innovation Management, v. 1, n. 1, p. 37-56, 2000.

ESCORSA, P.; VALLS, J. Tecnología e innovación en la empresa. Dirección y gestión. Barcelona: Ediciones UPC, 1998. 
ETZKOWITZ, H.; LEYDESDORFF, L. The Triple Helix University-lndustry-

Government Relatios: A Laboratory for Knowledge Based Economic Development. Theme paper, Amsterdan, 1995.

FREEMAN, C. Política de Tecnología y Desempeño Económico: Lecciones de Japón. London: Pinter Publishers, 1987.

The Economics of Technical Change. Camb. J. Econ., v. 18, n. 5, p. 463-514, 1998.

The National System of Innovation in Historical Perspective. Cambridge Journal of Economics, n. 19, 1995

GIBBONS, M.; LIMOGES, C. et al. The New Production of Knowledge: The Dynamics of Science and Research in Contemporary Societies. London: Sage, 1994.

JIMÉNEZ, M. E. Evolución de los paradigmas y modelos interpretativos del desarrollo regional. Serie Gestión Pública 13. CEPAL, Ilpes, 2001.

JOINT RESEARCH CENTRE. The European Commission's in-house science service. Disponible en: <http://www.jrc.es/home/report/spanish/articles/vol79/ITP1S796.htm>.

LANDAU, R. How competitiveness can be achieved: fostering economic growth and productivity. Technology and economics. Washington, D.C: National Academy Press, 1991.

LEYDESDORFF, L.; BESSELAAR, P. V. (Eds.). Evolutionary Economics and Chaos Theory: New directions in technology studies. London: Pinter, 1994.

LEYDESDORFF, L.; MARTINS, M. Triple Helix indicators of knowledge-based innovation systems. Research Policy, v. 35, 2006.

LIST, F. The National System of Political Economy. Translated by Sampson S. Lloyd. Fourth Book, “The Politics”. London: Longmans, Green and Co., 1909.

LOVE, J. H.; ROPER, S. Location and network effects on innovation success: evidence for UK, German and Irish manufacturing plants. Research Policy, p. 643-661, 2001

LUNDVALL, B. A. et al. National systems of production, innovation and competence building. Research Policy, v. 31, p. 213-231, 2002. 
MADOREY, O. EI territorio como factor estratégico de desarrollo. Hacia un espacio de gestión metropolitana en el Gran Rosario. Seminario Ciudad Futura. Rosario, Argentina, 1999.

MANUAL DE FRASCATI. Fuandación Española Ciencia y Tecnología. 2002.

Disponible en: <http://www.ocyt.org.co/Manual\%20de\%20Frascati\%202002.pdf>.

NELSON, R. Innovación. In: SILLS, D. L. (Dir.) Enciclopedia internacional de ciencias sociales. Madrid: Aguilar, 1976.

NELSON, R. R.; WINTER, S. G. In search of a useful theory of innovation. Research Policy, v. 6, n. 1, 1977.

NELSON, R. R.; WINTER, S. G.; LEVIN, V.; KLEVORICK, A. K.; GILBERT, R.; GRILICHES, Z. Appropriating the returns from industrial research and development. Brookings papers on economic activity, v. 18, n. 3, p. 783-832, 1987.

NONAKA, I.; TAKEUCHI, H. The knowledge-creating company. New York: Oxford University Press, 1995.

OECD. Libro verde sobre el espíritu empresarial en Europa. Comisión Europea. Dirección General de Empresa. Bruselas, 2003.

Manual de Oslo. Proposed Guidelines for collecting and interpreting technological innovation data. Paris: OECD, 1997.

PÉREZ-CASTAÑO, B. J. Apuntes sobre Innovación. Mimeo, Universidad del Valle, Cali, Colômbia, 2006.

Estrategias de competitividad basadas en la gestión del conocimiento para pymes manufactureras de Cali (Colombia). Tesis Doctoral. Universidad Politécnica de Valencia, España, 2001.

Sistema de Innovación para la Competitividad vinculando los Stakeholders. Sistema GUIES: Gobierno, Unidades de Investigación, Empresas y Sociedad. Working Paper Universidad Politécnica de Valencia, España, 2011.

PIKETTY, T. Capital in the Twenty-First Century. The Belknap Press of Harvard University Press, 2014. 
PORTA, J. D.; PÉREZ-CASTAÑO, B. J. BAIXAULI, I.; BAIXAULI, J. J. Modelo para la Gestión de Capital Intelectual de una Región: Una aproximación. 3er Congreso Internacional de Gestión del Conocimiento y de la Calidad. Bogotá, Colombia, 2005.

PORTER, M. E. Innovation: Location Matters. Sloan Management Review, v. 42, n. 4, 2001.

La ventaja competitiva de las naciones. Buenos Aires: Cecsa, 1990.

ROBERTS, E. Gestión de la Innovación tecnológica. Clásicos Cotec, n. 1, 1996.

ROGERS, E. M.; HALL, B. J.; HASHIMOTO, M.; STEFFENSEN, M.; SPEAKMAN, K. L.; TIMKO, M. K. Technology transfer from university based research centers: the University of New Mexico experience. Journal of Higher Education, v. 70, n. 6, p. 687-705, 1999.

ROGERS, E. M.; LARSEN, J. K. La fiebre del Silicon Valley. Barcelona: Reverté, 1986.

ROSENBERG, N. Inside the Black Box Technology and Economics. Nueva York: Cambridge University Press, 1982

SÁBATO, J.; BOTANA, N. La ciencia y la tecnología en el desarrollo futuro de América Latina. Revista de la Integración, n. 3, p. 15-36, 1968.

SAMSON, K. J.; GURDON, M. A. University scientist as entrepreneurs. A special case of Technology Transfer and high-tech venturing. Technovation, v. 13, n. 2, p. 63-71, 1993.

SCHUMPETER, J. A. The Theory of Economic Development. Cambridge: Harvard University Press, 1934; Nueva York: Oxford University Press, 1961. (First published in German, 1912.)

SMILOR, R. W.; GIBSON, D. V.; DIETRICH, G. B. University spin-out companies.Technology Starts ups from university of Texas at Austin. Journal of Business Venturing, v. 5, n. 1, p. 63-76, 1990. 
SOLOW, R. Challenge. In: ROSTRUP-NIELSEN, J. (Ed.) Política de innovación y relaciones universidad/industria. The IPTSReport, 2005.

STARFIELD, B. Improving equity in health: A research agenda. International Journal Services, v. 31, n. 3, p. 545-66, 2001.

STIGLITZ, J. E. Conferencia "Hacia una economía sustentable: conflicto y postconflicto en Colombia”. Bogotá, 2003.

La sociedad del aprendizaje. EI Espectador, 2014. Las crisis post crisis. El Espectador, 2013.

TUSHMAN, M. L.; MOORE, W. L. (Eds.) Readings in the Management of Innovation. Londres: HarperCollins, 1988.

VASCONCELOS, G.; WILKINSON, J.; AMANCIO, R. Empreendedorismo, inovacao e redes: uma nova abordagem. RAE-eletrônica, v. 7, n. 1, 2008.

VÁZQUEZ-BARQUERO, A. Desarrollo local. Una estrategia de creación de empleo, Madrid: Editorial Pirámide, 1988.

Recebimento dos originais: 01/05/2015

Aceitação para publicação: 27/08/2015 